2017-07-20

\title{
Soft robotic snake with variable stiffness actuation
}

Sheard, J

http://hdl.handle.net/10026.1/9979

10.1007/978-3-319-64107-2_29

Lecture Notes in Computer Science (including subseries Lecture Notes in Artificial Intelligence and Lecture Notes in Bioinformatics)

All content in PEARL is protected by copyright law. Author manuscripts are made available in accordance with publisher policies. Please cite only the published version using the details provided on the item record or document. In the absence of an open licence (e.g. Creative Commons), permissions for further reuse of content should be sought from the publisher or author. 


\title{
Soft Robotic Snake with Variable Stiffness Actuation
}

\author{
Ryan Draper $^{1}$, Jane Sheard ${ }^{1}$, Matt Troughton ${ }^{1}$ and Martin F. Stoelen ${ }^{2}$ \\ ${ }^{1}$ School of Computing, Electronics and Mathematics, Plymouth University, UK \\ ${ }^{2}$ Fieldwork Robotics Ltd, Plymouth, UK \\ ryan.draper@students.plymouth.ac.uk, jane.sheard@students.plymouth.ac.uk, matt.trough- \\ ton@students.plymouth.ac.uk, martin.stoelen@plymouth.ac.uk
}

\begin{abstract}
In this paper, we present a prototype of a 3D printed snake-like robot for search and rescue applications, inspired by biological snake anatomy and locomotion. Unlike traditional robotics, this design takes advantage of soft materials to create a robot that is resilient to shock impacts, such as from falling debris or unsound flooring, and that can very its stiffness. The robot uses a flexible spine to connect multiple sections, allowing controlled actuation while providing a sturdy structure. Variable stiffness actuation is implemented through the use of elastic materials to act as tendons for the body, in an agonist-antagonist setup. Actuation occurs through the use of Robotis Dynamixel AX-12A servos, controlled by a Trossen Robotics Arbotix-M Robocontroller. The design features a head, containing a Raspberry Pi 3 and a Pi Camera Module. This added embedded computation can connect to a remote PC via wireless communication, allowing an operator to control the robot. This paper discusses the design and early stage testing for the prototype, and shows that robots based on soft $3 \mathrm{D}$ printed materials and mechanisms are viable, and effective.
\end{abstract}

Keywords: Soft Robotics, Bio-Inspired Robotics, Locomotion, Variable Stiffness.
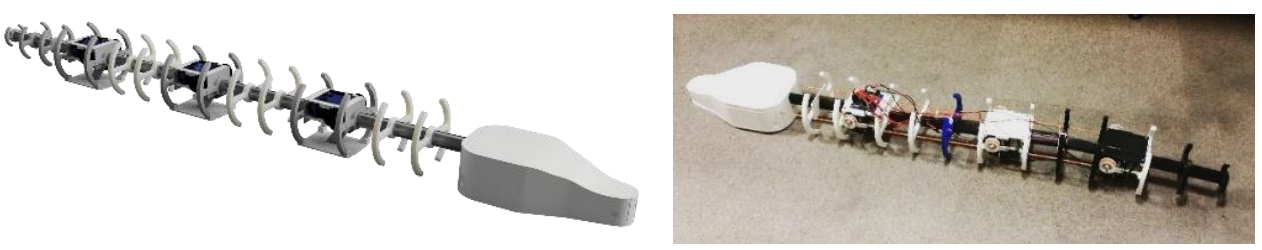

a) $3 \mathrm{D}$ rendering of the snake robot design.

b) Basic working prototype of the snake robot.

Fig. 1. The prototype variable-stiffness snake robot design

\footnotetext{
${ }^{1}$ MEng(hons) Robotics students, Plymouth University
} 


\section{Introduction}

Limbless creatures have evolved to use methods of locomotion that differ quite radically from methods seen in limbed creatures. However, these methods are just as effective when manoeuvring over uneven terrain or through small areas, which makes them an interesting and useful study for facilitation in search and rescue robotics.

Snake locomotion consists of various techniques, typically of a wave-like motion, where lateral movement and bending propagates from head to tail to propel the body. The methods of snake motion are serpentine, concertina, sidewinder, and rectilinear motion [1]. Of these locomotion methods, the sidewinding motion involves the snake moving itself laterally in an S-shape. This is typically used in areas where there are few resistance points for the snake to push its body forwards. The concertina motion involves folding the body into a compressed shape to move itself forwards in sections. This works well for tree climbing, as the snake can use its scales to grip the surface, bunch its body, and spring its head forwards to find a new grip surface. Rectilinear uses vertical contraction and extension of the body to create a ripple effect, which causes a slower method of motion. And lastly the serpentine motion, which is the creation of side to side contractions, and the most common method of locomotion seen in sea and land snakes. The snake's scales are used to push against resistance points, thrusting its body forwards [2].

Using soft materials, the robot can be designed to withstand impacts, as well as provide a flexible structure to the body. This allows the anatomy of a snake body to be recreated, creating a main spine to support the body, as well as providing the flexibility to bend. Ribs can be replicated to add protection to its internal assembly, and add impact resistance to the design by using a material with high toughness and resistance to deformation. Combining these, a prototype and early stage design for a soft robotic snake is created, tested and examined for search and rescue methods.

Implementing an agonist-antagonist muscle pair structure, with a flexible tendon material, variable stiffness actuation can be employed to control the robots stiffness and movement. This muscle pair works by contracting one muscle, while the other relaxes, to move a structure along a specific plane. By contracting both muscles at the same time, the joint can be held at a stable position. The design is currently only fully controlled in the horizontal plane, but could easily be expanded to be fully actuated. A fully actuated robot would function as a continuum-type design.

\section{Design Process}

\subsection{Mechanical Design}

One of the design goals was to use as few moving or fragile components as possible, creating a design as robust as possible in unsafe environments. To achieve this, a structure composed of a flexible spine supported by rigid ribs was designed. As the snake is only fully controlled in the horizontal plane, the spine is flexible in the horizontal direction and stiff along the vertical. The ribs are designed to act as a body, allowing the actuation of the spine to push against objects and generate motion. The ribs are also 
able to cushion sensitive components against impacts by partially flexing. The 3D model of the spine and rib structure can be seen in Fig. 2.

The body is intended to be moved using a form of soft agonist-antagonist actuation, rather than a structure based on wheeled motion [3]. An extensible fibre is used to transfer the servo actuation to the body. The servos are all of type Dynamixal AX-12 (Robotis, Korea). The tendons are made from a length of FilaFlex (Recreus, Spain) 3D printable filament, wrapped with nylon fishing wire. These filaments have close to quadratic response between load and extension, similar to biological tendons [4]. The tendon design was inspired by that used on the GummiArm variable-stiffness robot manipulator [5]. Each tendon is attached to a pulley, connected to one horizontal actuation servos, and to the rear of the next section ${ }^{3}$. Each section then has a tendon pulling in both horizontal directions, allowing control over the stiffness of the structure. Most current snake robots use mechanical joints or hinges rigidly connected to servo-motors to create movement. This is susceptible to damage that can render the joint immobile: either by direct deformation of a linkage, or by damage to the gears or motor due to the high effective inertia of the rigid joint. By using a flexible spine structure and elastic connections to the servos, the risk of damage that can reduce the robot mobility is greatly lessened.

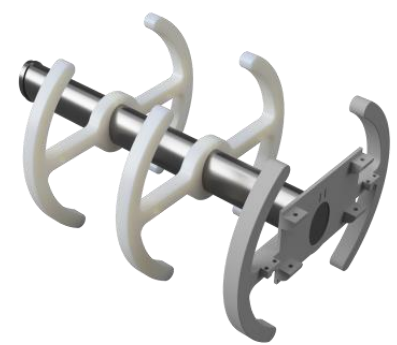

Fig. 2. 3D model of the body structure, showing the structure of the robot's ribs and the flexible spine

\subsection{Electrical Design}

The electrical system is designed to be resistant to damage and allow an easy addition of multiple sections. Every section is identical, except the main section immediately following the head. A single section can contain three servos, and a small $12 \mathrm{~V}$ $1100 \mathrm{mAh}$ Lithium-Polymer battery. The section following the head also holds an Arbotix-M micro-controller (Trossen Robotics, Downers Grove, Illinois, USA). The Arbotix-M is connected to each servo through a daisy chained TTL and power wire, allowing it to control the motion of every section. This is a software emulation of the central pattern generators found in Vertebrates, similar to a Central Pattern Generator found in vertebrates [6].

The batteries in each section are wired in parallel, and connect to an adjacent section. This design prevents the sections from individually running out of power. It also allows the possibility of partial retained functionality in the case of a section being severed; if an additional wireless radio is mounted on each section, and an Arbotix-M is present in

\footnotetext{
${ }^{3}$ Repository for the GummiArm robot project: https://github.com/mstoelen/GummiArm
} 
both sides of the severance, each portion can remain in contact and operational. The radio would then provide a method of wireless communication with this section, allowing an operator to control it.

The maximum current for each servo is $900 \mathrm{~mA}$, and the Arbotix-M can draw up to $500 \mathrm{~mA}$, therefore the minimum possible battery life is around 20 minutes, extended slightly with any added sections. This is shown in equation 1.

$$
\text { Lifetime }(\text { minutes })=\text { Sections } * 1 /(\text { Sections } * 0.9 * 3)+500
$$

It is likely that the servos will draw on average about one third maximum current, depending on stiffness settings. This leads to an average battery life of around 60 minutes, again increasing slightly with the number of sections. This is also shown in equation 2.

$$
\text { Lifetime }(\text { minutes })=\text { Sections } * 1 /(\text { Sections } * 0.3 * 3)+500
$$

Section position is monitored through the spine angle, measured with strain gauges (Spectra Symbol, Salt Lake City, Utah, USA) connected to the ribs and running along the top of the spine. The resistance of the gauges is measured by the Arbotix-M through the use of a potential divider. The Arbotix-M uses this data in a PI control algorithm to control the spine curvature [7].

\subsection{Control Unit Design}

The snake robot's head has been designed to include a Raspberry Pi 3 microcomputer to act as the robot main control centre, to interface with a camera, the servo controller, and to pass messages between the robot and the user. The robot can either use the Pi Camera Module v2 or the Pi NoIR Camera v2, depending on requirements. Both camera modules have a Sony IMX219 8MP sensor, which gives a 1080p image at $30 \mathrm{fps}$, but the NoIR Camera doesn't contain an infrared filter, so it allows night vision when used with infrared lighting or LED's.

For search and rescue applications, the robot will either be equipped with the Pi Camera and the option of regular LED's for lighting up dark areas, or the NoIR Camera with infrared lighting. For testing of the robot, the head has been designed using the Pi Camera without any LED's, as it will only be tested in well-lit conditions.

The Raspberry Pi 3 requires $5 \mathrm{~V}$ at $2.5 \mathrm{~A}$ for full power, so a method of internal power has been evaluated for use without main power. As the Raspberry Pi is only communicating with the camera module, the Arbotix-M via TTL, and currently no other peripherals, its current usage is estimated at about 750-1000mA. A 10Ah USB power pack was chosen, as it offers $5 \mathrm{~V}$ at $2.1 \mathrm{~A}$, which will satisfy this current usage.

Power usage of the Raspberry Pi:

$$
5 V * 1 A=5 W
$$

Power supply capacity from battery pack:

Run time for powering Raspberry Pi:

$$
5 \mathrm{~V} * 10 \mathrm{Ah}=50 \mathrm{Wh}
$$

$$
\frac{50 W h}{5 W}=10 \text { hours }
$$

Therefore, using this internal battery pack, the head system will be expected to run continuously for 10 hours between charges. Doubling the current usage of the Raspberry Pi to $2 \mathrm{~A}$, the head can still be supported by the battery pack, and provide power for 5 hours. 


\section{Implementation}

\subsection{Snake Body}

The snake body was designed using Autodesk Fusion360's parametric modelling system, which allowed quick creation and modification of the design as it progressed. By utilising Fused Filament Fabrication (FFF) 3D printing technology, it was possible to rapidly develop, test, and improve the designs.

The snake robot is built from multiple sections, consisting of servo mounts and spine structures. There is no limit to the amount of sections that can be implemented, but a head and the movement control section must be at the front of the robot.

Multiple variations of spine and printing methods were tested. It was found that for ease of 3D printing, and horizontal-only flexibility, the best shape for the spine was an ellipse. There are $1 \mathrm{~mm}$ thick ridges, separated by $78 \mathrm{~mm}$, along the structure. The ridges are for the placement of the ribs, and are designed to fit into the groove in the centre of the ribs.

Throughout the design, different spines were trialled with both 3 and 4 ribs, and tested using various materials and infill percentages. The results of these tests can be seen in experiment 4.1. The ideal spine in this experiment holds 3 ribs and would be printed at a low infill using NinjaFlex (NinjaTek, Manheim, PA, USA), however, in practice low infill spines have a strong tendency to buckle, causing non uniform bending and severely hampering both the strain sensors and PI control. For this reason, the most appropriate makeup of a spine is high infill NinjaFlex.

The ribs were similarly tested through tensile and compressive testing, as seen in experiment 4.3 and experiment 4.4. The rib material found to be most suitable was high infill SemiFlex (NinjaTek, Manheim, PA, USA) ribs, as it provided a good resistance to deformation, whilst not breaking at high strain. This allows the ribs to absorb the forces from an impact.

The servos providing horizontal movement are mounted between two boards that connect the two adjacent section spines, as seen in Fig. 3. The servos control the spine for the section in front of them, and wind the tendons upon actuation. The tendon materials were also evaluated for tensile strength, as seen in experiment 4.3. The nonlinear behaviour of the tendon allows for variable stiffness control of each section, accompanied with the use of the servos as an agonist-antagonist muscle pair [8].

An additional servo can be mounted on the underside of the servo mounting board. This servo can control a brake that provides additional grip, or a flexible connection to other servo mounts, allowing for some slight control in the vertical direction by curling the snake. In addition to this, a rough surface plate made of NinjaFlex can be mounted on the bottom of the servo mount. This plate grants additional friction, making movement more efficient. 


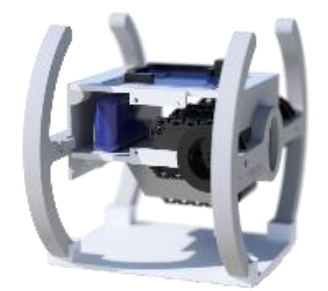

Fig. 3. 3D model of the servo mount section, displaying ribs either side, which attaches to the spine, servo mounting boards, servos, and underside servo brake and plate

\subsection{Movement Control}

The calculations for section position are handled by the Arbotix-M, which uses data from 2 variable resistance strain sensors attached to a spine. These sensors have a linear response in one direction, and a mostly-linear response in the other. The sensors are powered by $5 \mathrm{~V}$ from the Arbotix-M, and their voltage read by the Arbotix-M through a $20 \mathrm{~K}$ Ohm resistor, connected to Ground. The relationship between voltage and spine position must be determined for each spine section individually.

The Arbotix-M uses the determined position value as the control unit for the PI algorithm, which drives the servos to pull the spine to its required position. The PI outputs a desired torque and direction for each servo. The servos are configured in wheel mode, rather than position mode, as positional only allowed 270 degrees of movement. This was unpractical for the design, because the pulley diameter required to drive large movements with only 270 degrees of rotation would be quite large. As the spine itself acts as a third spring in the system, always pulling towards the centre, the torque required for greater angles does not increase linearly.

As the servos are torque controlled, the servo that is unspooling runs at a third of the rate of the spooling servo. This prevents the servo from unspooling too fast, and winding the tendon in the wrong direction. When the robot is within a few degrees of the target position, both motors will begin to tighten the tendons by winding them in. This allows the snake to resist being pushed off target, and ensures the tendons are always spooled correctly.

The robot is being designed to have four methods of locomotion, based on the real snake movement patterns studied [9]. The method of locomotion that the robot will attempt is determined by the user input from the control PC, and relayed to the ArbotixM. The possible locomotion methods recreate the 4 methods of snake motion: Serpentine, Concertina, Sidewinder, and Rectilinear motion. Serpentine motion is emulated through each section moving through a sinusoidal pattern, out of phase by 90 degrees. As true serpentine motion is not possible without axial and oblique actuation, this is only an approximation [10]. Concertina motion makes use of the $3^{\text {rd }}$ servo mounted underneath to control a brake. The brakes are placed down to generate additional friction with the floor, while either curling sections up or using them to push the body. Sidewinder motion also makes use of the brakes, by holding the snake in place while pushing the centre section to one side, before anchoring the centre and moving the front 
and rear sections in line. Rectilinear motion can be created by using a flexible connection between the bottom servos. Moving the servos in alternating directions causes the segments to curl alternately, creating a caterpillar like motion.

\subsection{Communication}

The Raspberry Pi in the snake head receives the images from the Pi Camera, and sends them to the operator PC, to be viewed by the user. The user can then control the robot with the keyboard buttons, which send commands back to the Raspberry Pi as a message, and further sends them to the Arbotix-M for servo control.

To implement this, the Raspberry Pi uses the Ubuntu operating system to allow the use of Robot Operating System (ROS) for sending messages. ROS provides a network, or communication, infrastructure, to send data between nodes, running multiple different processes in parallel. These nodes can be within the same computer, or across different computers, transferring data via Ethernet or Wi-Fi. Thus, messages can be sent from the Raspberry Pi to the PC, and vice versa, simultaneously. Fig. 4 outlines the overall communications throughout the robot.

The image messaging system was implemented to provide the user with a form of feedback from the movement mechanisms. After testing, the frame rate of communication between both computers was found to be approximately 5 seconds between each image, in comparison with 0.3 seconds between each frame when simply communicating with the Raspberry Pi itself. Other methods of streaming the camera images were evaluated, deciding on the use of the MJPG streamer module, which takes a live sequence of images and streams them to a web browser via HTTP.

The system for sending movement commands to the robot was tested using different methods. The first method involved sending character messages from the keyboard using ASCII keys. This method didn't work simultaneously upon key pressing events, and instead required the user to send each character as a separate command. However, this method allowed a wider range of functionality when controlling the robot, as more buttons (and hence commands) are available. This was chosen to allow more flexibility of motion control, by allowing a choice of type of motion, individual section control, and total body control.

The other method of control evaluated required the use of the ROS package key_teleop, which reads the PC arrow keys and maps them to a linear and angular velocity command, where up and down correspond to 0.8 and -0.5 linear motion respectively, and left and right correspond to 1.0 and -1.0 angular rotation respectively. This method provided less overall control of the robot, as it only allowed basic forwards, backwards, left and right commands; but it was the simplest method for user friendliness, as well as working simultaneously with each button press, and could be used for total motion control of the robot. 


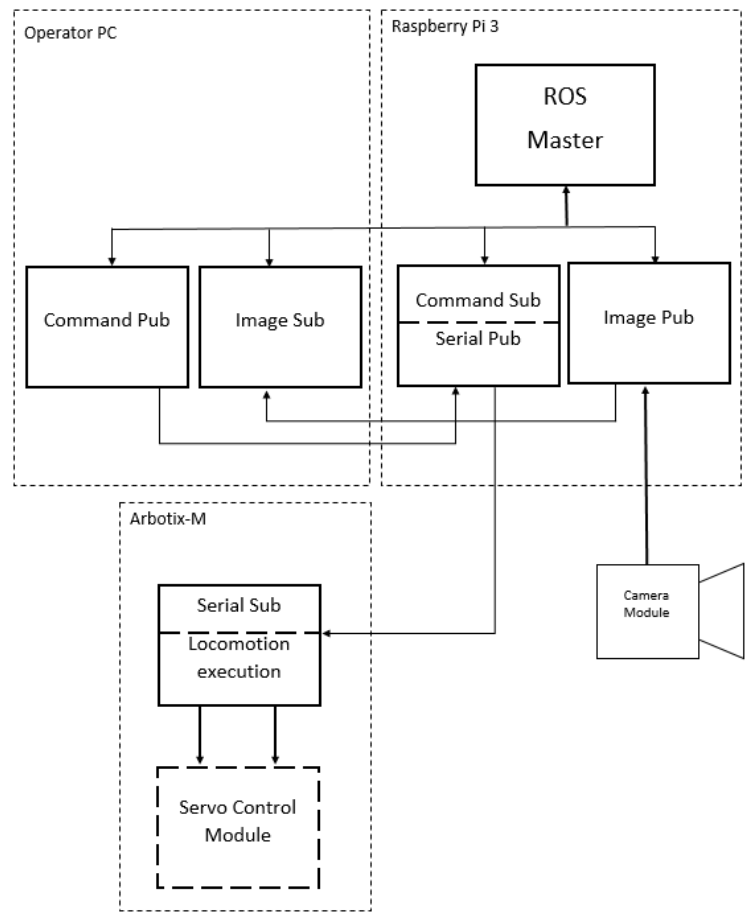

Fig. 4. Communication overview between the Operator PC, Raspberry Pi and Arbotix-M

The commands, from either method, are then published via ROS to a topic from the operator PC. The Raspberry Pi subscribes to the same topic, and receives these commands - either as velocity or string messages. The Raspberry Pi then communicates with the Arbotix-M via serial, sending corresponding messages to call the required locomotion function to control the body.

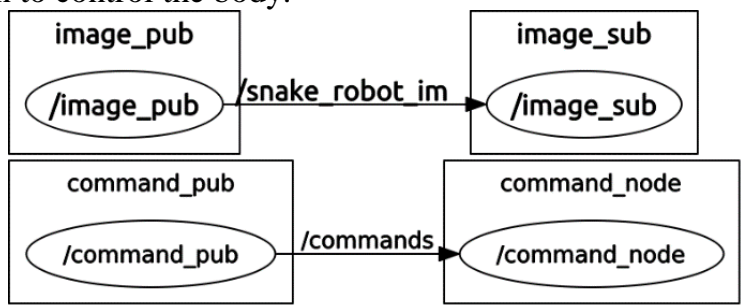

Fig. 5. RQT graph showing the ROS system in terms of nodes, topics and data flow

Fig. 5 shows the data flow using ROS, indicating the 2 separate processes running in parallel. The first sending messages from the Raspberry Pi to the PC, and the second sending commands back to the Raspberry Pi. This shows how the 2 systems run without interfering with each other, with improves the functionality of the system, allowing 
continuous processing rather than running sequentially. The project code is published open source on GitHub ${ }^{4}$.

\section{Experiments}

\subsection{Stiffness Testing}

Stiffness tests were conducted on various spine structures at different values of motor torque. The snake section was placed on the edge of a surface, and a $1 \mathrm{~kg}$ mass hung from the end. Deflection from 90 degrees was measured at different levels of servo torque. Both servos were set to wind in, to stiffen the snake body.

Fig. 6 shows that the SemiFlex spine is the most resistant to bending, as its deflection is typically lowest, but also has the smallest change in deflection with torque, suggesting it is too stiff. The low infill NinjaFlex spine is the least stiff, as it deflects the most, with the greatest sensitivity to change from little motor torque input. High infill NinjaFlex has the most linear response and also a good range of stiffness.

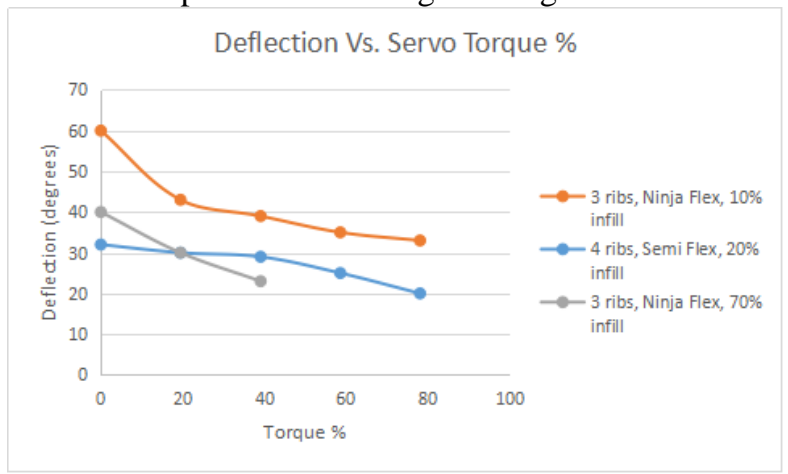

Fig. 6. Stiffness analysis of spines fabricated from different materials, evaluating spine deflection at various motor torques

From these results, the ideal spine appears to be low infill NinjaFlex, due to the low power required to move it and its large range of deflection available. However, a low infill material will be prone to buckling, therefore the high infill NinjaFlex was used for the robot spine structure due to its stiffness and deflection range.

\subsection{Finite Element Analysis (FEA) Testing}

FEA analysis was carried out on the 3D modelled rib structure, as these components receive the greatest load bearing. This analysis was carried out with three different possible materials: acrylonitrile butadiene styrene (ABS) plastic, polylactic acid (PLA), and Nylon. This analysis was done in Autodesk Fusion360's simulate workspace.

\footnotetext{
${ }^{4}$ Project on GitHub for full code development: https://github.com/JaneSheard/roco504

See also channel on YouTube for videos of testing and first attempts at locomotion: https://www.youtube.com/channel/UC-NMGpYUNjFGybRwE0-Zjaw
} 
These materials were selected for virtual testing as options for 3D printing, whilst flexible materials such as SemiFlex and NinjaFlex were tested practically in other methods, as discussed in experiments 4.3 and 4.4.

The ribs were constrained at the contact point with the floor, and a force of $10 \mathrm{~N}$ was placed on the servo board mounting points. The results of the test can be seen Fig. 7. It is noticeable that ABS has deformed the most, followed by Nylon and finally PLA.

The results from this analysis show that PLA would be the most appropriate material to use, however this test may not be accurate. The model has deformed by a significant percentage, which may have cause inaccuracies. Additionally, the simulation does not take into account the anisotropic properties of 3D printed materials, likely leading to over-estimated strength.

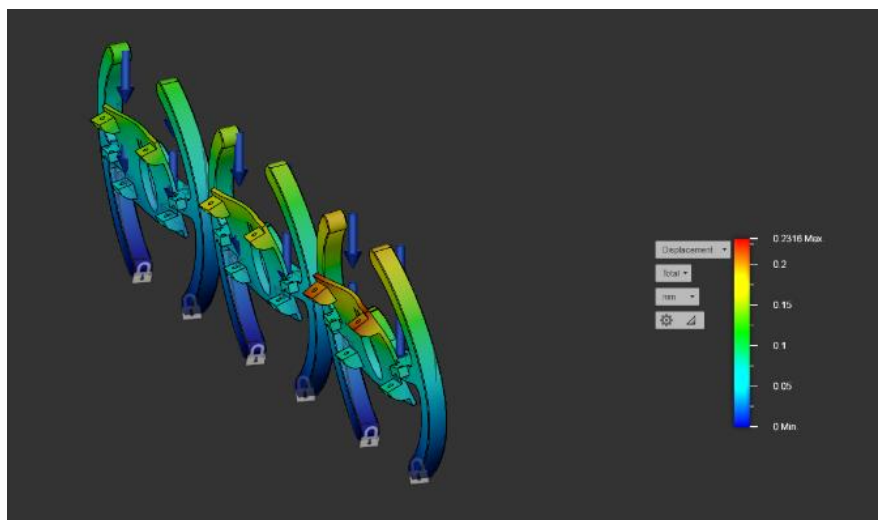

Fig. 7. FEA analysis of the 3 rib materials: PLA, Nylon and ABS, respectively

\subsection{Tensile Testing}

Tensile testing was performed on 3 ribs of different materials: PLA, 70\% infill SemiFlex and $80 \%$ infill NinjaFlex. The ribs were tested at $10 \mathrm{~mm} / \mathrm{minute}$, held lengthways and constrained just below the arcs.

Fig. 8 shows the results from tensile testing 3 types of rib, displaying PLA, SemiFlex and NinjaFlex.

PLA extended only $9 \mathrm{~mm}$ before failure, with a steep gradient in the elastic region before reaching its elastic limit, which shows it has a high stiffness. SemiFlex extended $317 \mathrm{~mm}$ before breaking, with a larger elastic region, high Ultimate Tensile Strength (UTS), and greater flexibility than PLA. NinjaFlex failed in completing the test, as the material was too flexible to be pulled, falling out of the machine grips. It remained in its elastic region throughout its testing, displaying a low Young's Modulus.

This test shows that high infill SemiFlex is the most appropriate rib material, due to its large elasticity and high toughness. The yield strength is very high, at over 1100 Newtons, equivalent to a $110 \mathrm{~kg}$ weight. This is larger than any tensile weight likely to occur, providing a large safety factor. 
Tensile Testing of Different 3D Printed Materials

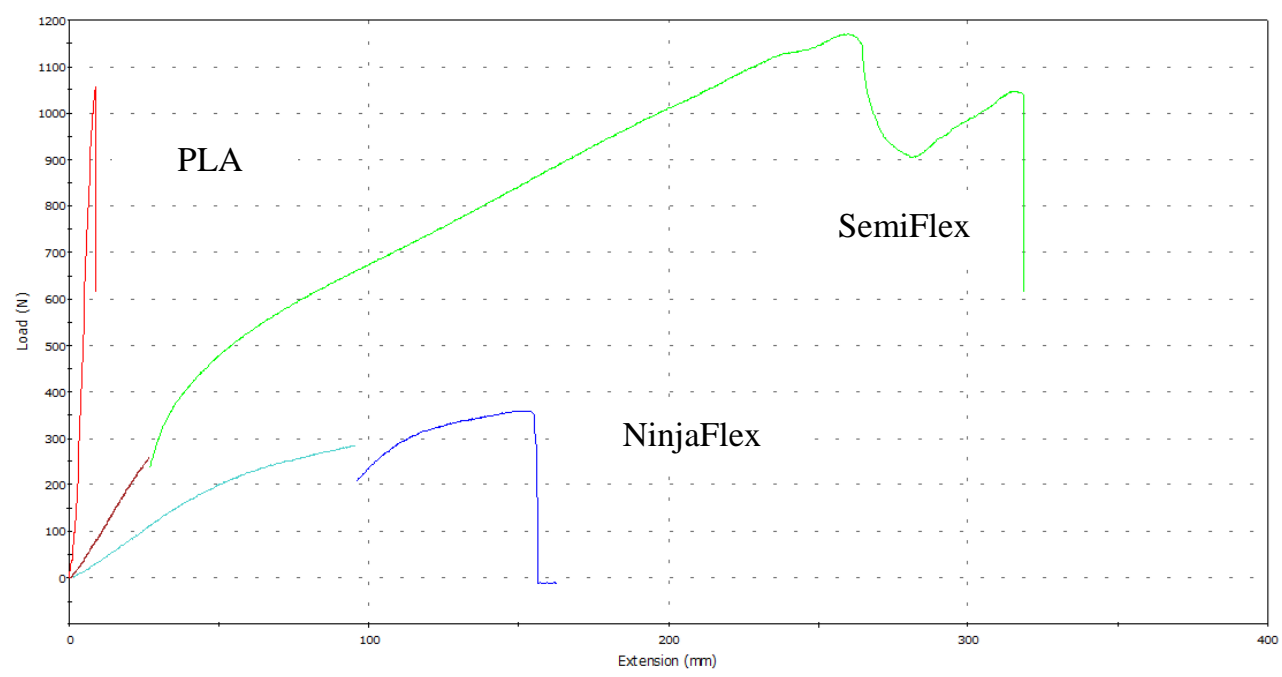

Fig. 8. Tensile testing different 3D printed materials for the rib structure: PLA, SemiFlex and NinjaFlex, respectively

Two tendons were also tested for tensile strength, as seen in Fig. 9. The tendons were identical except for the pitch of the coiled nylon thread. The tendons were tested at a rate of $100 \mathrm{~mm} /$ minute and were pre-loaded with 1 Newton.

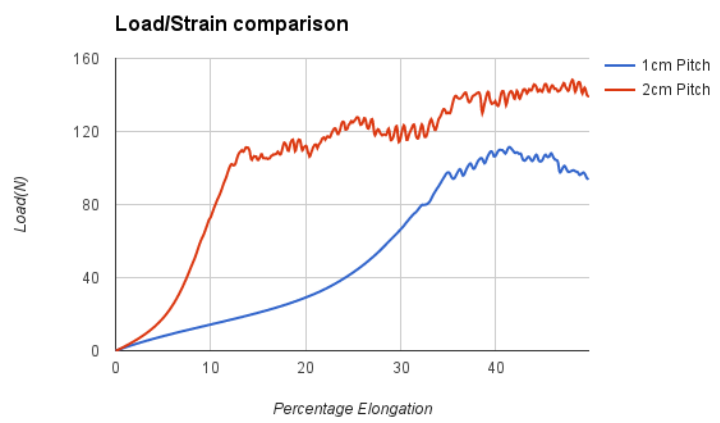

Fig. 9. Tensile testing tendon structures, evaluating different nylon thread pitch

Each tendon eventually failed as the wound nylon detached from the FilaFlex. It can be seen that the $1 \mathrm{~cm}$ pitch tendon extended much further than the $2 \mathrm{~cm}$ pitch before failing, with less load. This shows that smaller pitch leads to a more extensible tendon. 

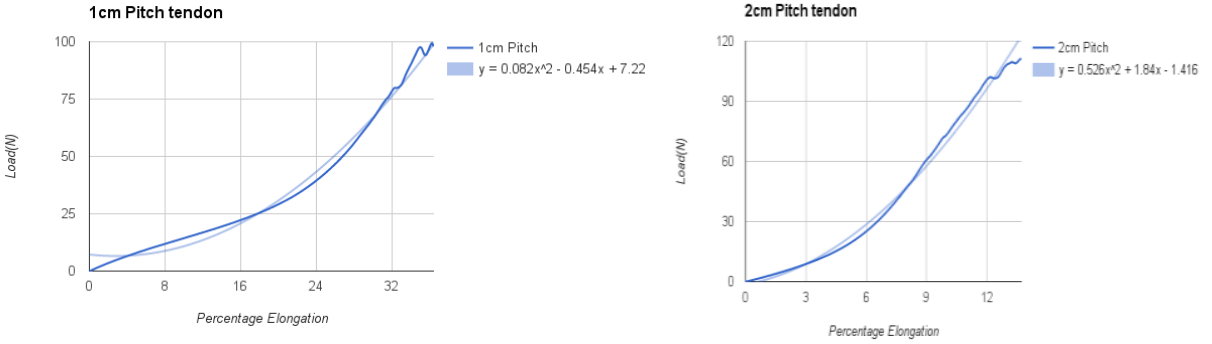

Fig. 10. Analysis of load-strain relationship of tendons with different nylon thread pitch

It can also be seen in Fig. 10, that the $1 \mathrm{~cm}$ pitch tendon has a load-strain relationship much closer to a quadratic. This would make the $1 \mathrm{~cm}$ pitch tendon more suitable for a variable stiffness system, by making exclusive control of stiffness and position more easily possible.

\subsection{Compressive Testing}

Compressive testing was also performed on the ribs to analyse their impact potential. Three rib terminators were used, PLA, 20\% infill NinjaFlex, and 80\% infill NinjaFlex. These samples were compressed at $50 \mathrm{~mm} /$ minute, and were preloaded with 1 newton. The NinjaFlex ribs were tested 3 times to ensure correct results. The PLA rib failed in its first test.

It can be seen in Fig. 11 and Fig. 12, that the $80 \%$ NinjaFlex rib is much tougher than the $20 \%$. It also maintained its structure far better and distributed load more evenly. The PLA rib, in Fig. 13, required a large amount of force to fail, but failed in a brittle fashion, with very little compression.

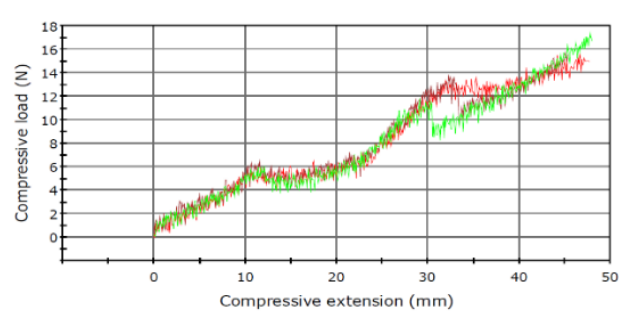

Fig. 11. Compressive testing $20 \%$ infill NinjaFlex

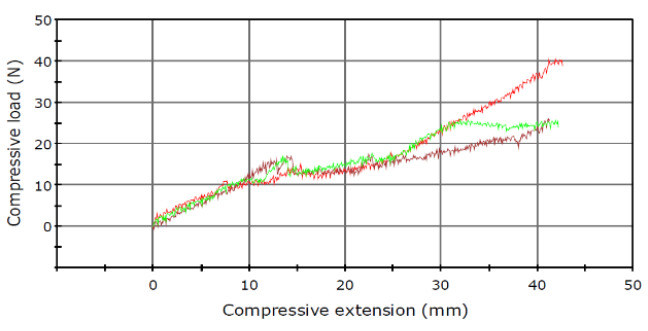

Fig. 12. Compressive testing $80 \%$ infill NinjaFlex 


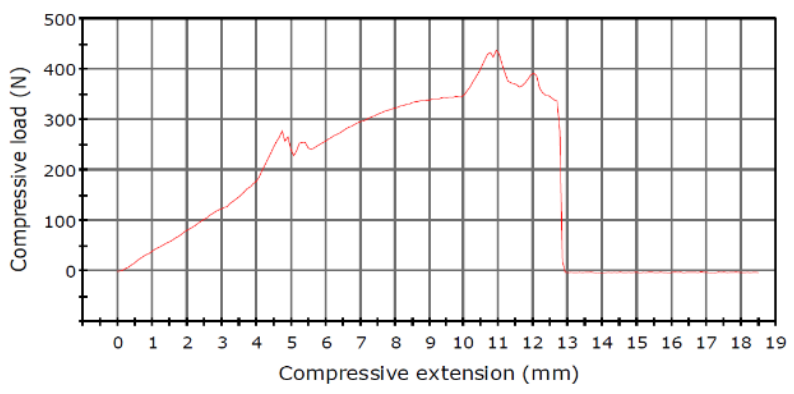

Fig. 13. Compressive testing $20 \%$ infill PLA

From these tests, it is clear that either a high infill SemiFlex or NinjaFlex material would be suitable for the rib structure, with a high infill SemiFlex being chosen due to its high toughness and flexibility, whilst maintaining its structural integrity.

\section{Discussion}

Extensions to this project focus on increasing its functionality for search and rescue missions. The addition of artificial intelligence approaches would give the robot more autonomous functionality for search and rescue. This could be implemented by developing camera processing, to understand its environment, and including a thermal imaging camera for locating humans in rubble.

Extra hardware would be beneficial to the system, such as microphones to detect any possible noise, and to transmit these readings to the robot user. A method of navigation would aid the system, by adding a GPS and a 3D simulated model of the path taken during search missions. This would create a simple method for the snake to follow its own path, and for easy localisation of the robot by the user.

Current-controlled DC motors would likely be a more efficient actuation method, as they do not require as much space and would allow for more degrees of control, and a smaller total size. It may be that an alternative actuation system to motors altogether would be preferable.

The locomotion of this prototype is very slow. A majority of the energy expenditure by the robot is wasted as it cannot maintain grip on most surfaces. Using the serpentine method, the sections tended to slip horizontally rather than provide thrust. Average travel speed was around $1 \mathrm{~cm}$ per minute. Concertina and Sidewinder both suffered a similar problem, and averaged a travel speed of near $2 \mathrm{~cm}$ per minute. Rectilinear was the most effective form of movement, due to a portion of the robot weight being lifted from the floor and all motion occurring in the plane of the desired forward direction.

To ensure reliable and efficient propulsion of the robot, extra degrees of control need to be added. Particularly important is the addition of oblique actuation, allowing the snake to twist and push against the ground more efficiently, similarly to a biological snake. Adding a flexible skin, with external pressure sensing, to the robot body would create a smoother surface when moving through rubble, provide a surface of friction to aid thrust, and allow the robot to react to tight spaces and collisions on its body [11]. Further to this, the lower the centre of gravity of the body, the better it will perform for 
snake-like locomotion, so a lower placement of the control servos would be recommended in a future design [12].

\section{Conclusion}

In this paper, a first prototype of a snake with flexible, and variable-stiffness, joints have been presented. Such joints have several advantages over traditional rigid joints: they are more resilient to damage from sudden impacts, and can conform to the environment. Full control over the stiffness is possible through implementation of an agonist-antagonist system, with actuation through custom-made soft tendons driven by servos. The implementation of a spine structure can also be expanded to an omnidirectional joint, or with many in series could be used to develop a continuum structure.

This paper also shows that 3D printing methods can be used to rapidly prototype a soft robot design. The tests show that soft 3D printed materials can perform remarkably well already, and they will inevitably improve. With improvements in 3D printing technology, the quality and complexity of components will also increase. It can also be used to rapidly create replacements for any field robot.

The snake robot is still somewhat inefficient in locomotion, but we believe that including more degrees of freedom and improved actuation is possible. This could result in a soft snake robot with interesting properties for real-world tasks, like search and rescue.

\section{References}

[1] The Mechanism of Locomotion in Snakes. Gray. J (1946) Journal of Experimental Biology 23:101-120.

[2] The mechanics of slithering locomotion. David L. Hu. Proceedings of the National Academy of Sciences of the United States of America, vol. 106 no. 25 10081-10085, doi: 10.1073/pnas.0812533106

[3] Loop forming snake-like robot ACM_R7 and its Serpenoid Oval control, T. Ohashi, H. Yamada and S. Hirose (2010) International Conference on Intelligent Robots and Systems (IROS) pp 413-418. doi: 10.1109/IROS.2010.5651467

[4] Massoud EIE. Healing of subcutaneous tendons: Influence of the mechanical environment at the suture line on the healing process. World Journal of Orthopedics (2013); 4(4): 229-240. doi: 10.5312/WJO.v4.i4.229

[5] Stoelen, M.F., Bonsignorio, F. and Cangelosi, A. Co-Exploring Actuator Antagonism and Bio-Inspired Control in a Printable Robot Arm, 14th International Conference on the Simulation of Adaptive Behaviour (SAB2016). Aberystwyth, UK. August, 2016, pp. 244-255.

[6] Hooper, Scott L (1999-2010). "Central Pattern Generators". Encyclopaedia of Life Sciences. John Wiley \& Sons

[7] Parameterized and Scripted Gaits for Modular Snake Robots. Tesch M (2012) Advanced Robotics vol.23 pp 1131-1158 doi: 10.1163/156855309X452566 
[8] Design and Architecture of a Series Elastic Snake Robot. D. Rollinson (2014) IEEE International Conference on Intelligent Robots and Systems pp 4630-4636 doi: 10.1109/IROS.2014.6943219

[9] Actuators. Coupling between Output Force and Stiffness in Different Variable Stiffness Actuators. Jafari, A (2014); MDPI 3(3): 270-284. doi: 10.3390/act3030270

[10] Design and improved serpentine curve locomotion control of a planar modular snake, $P$. Shi, Q. Shao and D. Liang (2016). International Conference on Information and Automation (ICIA) pp. 1398-1402. doi: 10.1109/ICInfA.2016.7832038

[11] Design of a modular snake robot. C. Wright (2007) International Conference on Intelligent Robot Systems pp. 2609-2614. doi: 10.1109/IROS.2007.4399617

[12] Design improvements and dynamic characterization on fluid elastomer actuators for a soft robotic snake. M. Luo, W. Tao, F. Chen, T. K. Khuu, S. Ozel and C. D. Onal (2014) International Conference on Technologies for Practical Robot Applications (TePRA) pp. 1-6. doi: 10.1109/TePRA.2014.6869154 